\title{
MODIFIED MODULUS OF SMOOTHNESS AND APPROXIMATION IN WEIGHTED LORENTZ SPACES BY BOREL AND EULER MEANS
}

\begin{abstract}
Using one-sided Steklov means, we introduce a new modulus of smoothness in weighted Lorentz spaces. The direct and inverse approximation theorem for this modulus of smoothness are proved. Also, we estimate the rate of approximation by the Borel and Euler means in weighted Lorentz spaces.
\end{abstract}

Key words: weighted Lorentz spaces, direct and inverse approximation theorems, Borel means, Euler means

2010 Mathematical Subject Classification: 42A10, 42A24

1. Introduction. Let $f$ be a $2 \pi$-periodic continuous function ( $f \in C_{2 \pi}$ ), $T_{n}$ be the space of trigonometric polynomilas of degree at most $n, n \in \mathbb{Z}_{+}=\{0,1, \ldots\},\|f\|_{\infty}=\max _{x \in[0,2 \pi]}|f(x)|$. Let us consider the best approximation $E_{n}(f)_{\infty}=\inf \left\{\left\|f-t_{n}\right\|_{\infty}: t_{n} \in T_{n}\right\}, n \in \mathbb{Z}_{+}$, and the modulus of continuity $\omega(f, \delta)=\sup _{0 \leqslant h \leqslant \delta}\|f(\cdot+h)-f(\cdot)\|_{\infty}$. Then the classical Jackson theorem states that

$$
E_{n}(f)_{\infty} \leqslant C \omega\left(f,(n+1)^{-1}\right), \quad n \in \mathbb{Z}_{+},
$$

while the inverse Salem-Stechkin inequality gives

$$
\omega(f, 1 / n) \leqslant C n^{-1} \sum_{k=0}^{n-1} E_{k}(f)_{\infty}, \quad n \in \mathbb{N}=\{1,2, \ldots\}
$$

(see $[6$, Ch. 7]). For a $2 \pi$-periodic locally integrable function $f$, we can consider two variants of Steklov means:

$$
s_{h}(f)(x)=h^{-1} \int_{x}^{x+h} f(u) d u, \quad s_{h}^{(2)}(f)(x)=(2 h)^{-1} \int_{x-h}^{x+h} f(u) d u .
$$

(C) Petrozavodsk State University, 2021 
Israfilov, Kokilashvili, and Samko [9] introduced a modulus of smoothness in a weighted Lebesgue space with variable exponent $L_{w}^{p(\cdot)}$ of order $r \in \mathbb{N}$

$$
\Omega_{r}^{*}(f, \delta)_{L_{w}^{p}}=\sup _{0 \leqslant h_{i} \leqslant \delta}\left\|\prod_{i=1}^{r}\left(I-s_{h_{i}}^{(2)}\right)(f)\right\|_{L_{w}^{p}},
$$

where $I$ is the identical operator, and obtained a Jackson-type estimate $E_{n}(f)_{L_{w}^{p}} \leqslant C \Omega_{r}^{*}(f, 1 / n)_{L_{w}^{p}}, n \in \mathbb{N}$, and the inverse result. For another modulus of smoothness, direct and inverse approximation theorems were obtained by Ky [13]. Many mathematicians, such as Akgün, Guven, Israfilov, Kokilashvili, Yildirir, studied the approximation by trigonometric polynomials in various weighted spaces. We note only the papers [17], [10] studying the moduli of smoothness defined with help of $s_{h}(f)$ and the papers connected with Lorentz spaces: [12], [20], [21], [1], [2].

2. Definitions. A Lebesgue measurable $2 \pi$-periodic function $w:[0,2 \pi] \rightarrow[0, \infty)$ is called a weight function if $w^{-1}(\{0\})$ has measure zero. If $w(E)=\int_{E} w(x) d x$ for a measurable subset $E$ of $[0,2 \pi]$, then

$$
f_{w}^{*}(t)=\inf \{\lambda \geqslant 0: w(\{x \in[0,2 \pi]:|f(x)|>\lambda\}) \leqslant t\} .
$$

Let $1<p, q<\infty, w$ be a weight. A measurable function $f$ on $[0,2 \pi]$ belongs to the weighted Lorentz space $L_{w}^{p, q}$, if

$$
\|f\|_{p, q, w}=\left(\int_{0}^{2 \pi}\left(f^{* *}(t)\right)^{q} t^{q / p-1} d t\right)^{1 / q}<\infty, \quad f^{* *}(t)=t^{-1} \int_{0}^{t} f_{w}^{*}(u) d u .
$$

The classical Lorentz spaces were introduced by Lorentz (see [15]). If $p=q$, then $L_{w}^{p q}$ coincides with the weighted Lebesgue space $L_{w}^{p}$.

A weight $w$ belongs to the Muckenhoupt class $A_{p}(\mathbb{T}), 1<p<\infty$, if

$$
|w|_{A_{p}}=\sup \left(|I|^{-1} \int_{I} w(x) d x\right)\left(|I|^{-1} \int_{I} w^{1-p^{\prime}}(x) d x\right)^{p-1}<\infty,
$$

where $p^{\prime}=p /(p-1)$ and the supremum is taken with respect to all intervals $I \subset \mathbb{R}$ whose length $|I|$ does not exceed $2 \pi$ (see [16]).

If $w \in A_{p}(\mathbb{T}), 1<p, q<\infty$, then the Hardy-Littlewood maximal operator is bounded in $L_{w}^{p, q}$ (see [5]). As a consequence, the operators $s_{h}$ 
and $s_{h}^{(2)}$ are uniformly bounded in $L_{w}^{p, q}$. Now, we define, for $r \in \mathbb{N}$, the following modulus of smoothness:

$$
\Omega_{r}(f, \delta)_{p, q, w}=\sup _{0 \leqslant h_{i} \leqslant \delta, i=1, \ldots, r}\left\|\prod_{i=1}^{r}\left(I-s_{h_{i}}\right)(f)\right\|_{p, q, w}
$$

It is clear that $\Omega_{r}(f, \delta)_{p, q, w}$ is finite for $w \in A_{p}(\mathbb{T})$ and $f \in L_{w}^{p, q}$. In [12] and [1], the authors consider another modulus of smoothness $\Omega_{r}^{*}(f, \delta)_{p, q, w}$, where operators $s_{h_{i}}$ in (1) are substituted by $s_{h_{i}}^{(2)}$. By definition, $E_{n}(f)_{p, q, w}=\inf _{t_{n} \in T_{n}}\left\|f-t_{n}\right\|_{p, q, w}, n \in \mathbb{Z}_{+}$. For $r \in \mathbb{N}, 1<p, q<\infty$, and $w \in A_{p}(\mathbb{T})$ we denote by $W_{p, q, w}^{r}$ the collection of all absolutely continuous on each period functions $f\left(f \in A C_{2 \pi}\right)$, such that $f^{\prime}, \ldots, f^{(r-1)} \in A C_{2 \pi}$ and $f^{(r)} \in L_{w}^{p, q}$.

For a function $f \in L_{w}^{p, q}$ and $r \in \mathbb{N}$, we define the Peetre's $K$-functiomal by

$$
K\left(f, t, L_{w}^{p, q}, W_{p, q, w}^{r}\right)=\inf _{g \in W_{p, q, w}^{r}}\left\{\|f-g\|_{p, q, w}+t\left\|g^{(r)}\right\|_{p, q, w}\right\} .
$$

If $1<p, q<\infty, w \in A_{p}(\mathbb{T})$, then $L_{w}^{p, q} \subset L_{2 \pi}^{1}:=L_{w_{0}}^{1,1}, w_{0}(x) \equiv 1$, (see the proof of Proposition 3.3 in [12]) and $f \in L_{w}^{p, q}$ has the Fourier series

$$
a_{0}(f) / 2+\sum_{k=1}^{\infty}\left(a_{k}(f) \cos k x+b_{k}(f) \sin k x\right)=: \sum_{k=0}^{\infty} A_{k}(f)(x) .
$$

Let us consider partial sums $S_{n}(f)(x)=\sum_{k=0}^{n} A_{k}(f)(x)$, the Borel means

$$
B_{r}(f)(x)=e^{-r} \sum_{k=0}^{\infty} r^{k} S_{k}(t) / k !, \quad r>0,
$$

and the Euler means

$$
e_{n}^{t}(f)(x)=(1+t)^{-n} \sum_{k=0}^{n}\left(\begin{array}{l}
n \\
k
\end{array}\right) t^{n-k} S_{k}(f)(x), \quad t>0, \quad n \in \mathbb{N} .
$$

More about these means can be found in the monograph by Hardy [7]. It is well known that for $f \in L_{2 \pi}^{1}$, the following limit

$$
\widetilde{f}(x)=(2 \pi)^{-1} \lim _{t \rightarrow 0+0} \int_{t}^{\pi}(f(x-u)-f(x+u)) \operatorname{ctg}(u / 2) d u
$$


exists a. e. on $\mathbb{R}$ (see [3, Ch. VIII, $\S 7]$ ). The function $\widetilde{f}(x)$ is called the conjugate function to $f$. If $\tilde{f} \in L_{2 \pi}^{1}$, then its Fourier series has the form $\sum_{k=1}^{\infty}\left(a_{k}(f) \sin k x-b_{k}(f) \cos k x\right)$.

\section{Auxiliary propositions.}

Lemma 1. Let $1<p, q<\infty, w \in A_{p}(\mathbb{T})$. Then the conjugation operator is bounded in $L_{w}^{p, q}$ and the inequalities

$$
\left\|S_{n}(f)\right\|_{p, q, w} \leqslant C_{1}\|f\|_{p, q, w}, \quad\left\|f-S_{n}(f)\right\|_{p . q, w} \leqslant\left(C_{1}+1\right) E_{n}(f)_{p, q, w},
$$

hold for $n \in \mathbb{Z}_{+}$and $f \in L_{w}^{p, q}$.

The statement concerning conjugation operator can be found in [11, Ch. 6, Theorem 6.6.2], while the inequalities (2) can be proved as in [3, Ch. VIII, §20].

Lemma 2 is stated in [20] for arbitrary $r>0$ with a reference to the method of Ky [14]. We give another proof for $r \in \mathbb{N}$.

Lemma 2. Let $1<p, q<\infty, w \in A_{p}(\mathbb{T}), t_{n} \in T_{n}, n \in \mathbb{N}, r \in \mathbb{N}$. Then

$$
\left\|t_{n}^{(r)}\right\|_{p, q, w} \leqslant C n^{r}\left\|t_{n}\right\|_{p, q, w}
$$

holds.

Proof. It is sufficient to prove (3) in the case $r=1$. Note that for $t_{n}(x)=\sum_{k=0}^{n}\left(c_{k} \cos k x+d_{k} \sin k x\right)$ we have

$$
t_{n}^{\prime}(x)=-\sum_{k=1}^{n} k\left(S_{k}\left(\widetilde{t_{n}}\right)(x)-S_{k-1}\left(\widetilde{t_{n}}\right)(x)\right)=\sum_{k=1}^{n-1} S_{k}\left(\widetilde{t_{n}}\right)(x)-n \widetilde{t_{n}}(x) .
$$

Since the operators $S_{k}$ are uniformly bounded in $L_{w}^{p, q}$ and $\left\|\widetilde{t_{n}}\right\|_{p, q, w} \leqslant$ $\leqslant C_{1}\left\|t_{n}\right\|_{p, q, w}$, we obtain

$$
\left\|t_{n}^{\prime}\right\|_{p, q, w} \leqslant C_{2}(n-1)\left\|\widetilde{t_{n}}\right\|_{p, q, w}+n\left\|\widetilde{t_{n}}\right\|_{p, q, w} \leqslant C_{1}\left(C_{2}+1\right) n\left\|t_{n}\right\|_{p, q, w} .
$$

Lemma 3 is proved in [1] also for $r>0$.

Lemma 3. Let $1<p, q<\infty, w \in A_{p}(\mathbb{T}), r \in \mathbb{N}, f \in W_{p, q, w}^{r}$. Then

$$
E_{n}(f)_{p, q, w} \leqslant C(n+1)^{-r} E_{n}\left(f^{(r)}\right)_{p, q, w} \leqslant C(n+1)^{-r}\left\|f^{(r)}\right\|_{p, q, w}, \quad n \in \mathbb{Z}_{+} .
$$


Lemma 4 is proved in [12, Proposition 3.2].

Lemma 4. Let $1<p, q<\infty, w \in A_{p}(\mathbb{T})$ and $\varphi(x, y)$ is a measurable $2 \pi$-periodic in each variable function. Then

$$
\left\|\int_{0}^{2 \pi} \varphi(x, \cdot) d x\right\|_{p, q, w} \leqslant \int_{0}^{2 \pi}\|\varphi(x, \cdot)\|_{p, q, w} d x .
$$

Lemma 5. Let $1<p, q<\infty, w \in A_{p}(\mathbb{T}), r, k \in \mathbb{N}, f \in W_{p, q, w}^{r}$. Then

$$
\begin{gathered}
\Omega_{r+k}(f, \delta)_{p, q, w} \leqslant C \delta^{k} \Omega_{r}\left(f^{(k)}, \delta\right)_{p, q, w}, \quad \delta \in[0,2 \pi], \\
\Omega_{k}(f, \delta)_{p, q, w} \leqslant C\left\|f^{(k)}\right\|_{p, q, w} \delta^{k}, \quad \delta \in[0,2 \pi] .
\end{gathered}
$$

Proof. It is sufficient to prove the first inequality of the Lemma for $k=1$. Let $0 \leqslant h_{i} \leqslant \delta, 1 \leqslant i \leqslant r+1, f \in W_{p, q, w}^{1}$ and $g(x)=\prod_{i=2}^{r+1}\left(I-s_{h_{i}}\right)(f)(x)$. Then we have

$$
\prod_{i=1}^{r+1}\left(I-s_{h_{i}}\right)(f)(x)=-h_{1}^{-1} \int_{0}^{h_{1}} \int_{0}^{t} g^{\prime}(x+s) d s d t .
$$

By Lemma 4 and the uniform boundedness of $s_{h}$ in $L_{w}^{p, q}$, we obtain

$$
\begin{aligned}
& \left\|\prod_{i=1}^{r+1}\left(I-s_{h_{i}}\right)(f)\right\|_{p, q, w} \leqslant C_{1} h_{1}^{-1} \int_{0}^{h_{1}} t\left\|t^{-1} \int_{0}^{t} g^{\prime}(\cdot+s) d s\right\|_{p, q, w} d t \leqslant \\
& \leqslant C_{2} h_{1}^{-1}\left\|g^{\prime}\right\|_{p, q, w} \int_{0}^{h_{1}} t d t \leqslant 2^{-1} C_{2} h_{1}\left\|g^{\prime}\right\|_{p, q, w} \leqslant 2^{-1} C_{2} \delta\left\|g^{\prime}\right\|_{p, q, w} .
\end{aligned}
$$

It is clear that $g^{\prime}=\prod_{i=2}^{r+1}\left(I-s_{h_{i}}\right)\left(f^{\prime}\right)$ a. e. on $\mathbb{R}$. Taking the supremum in the left-hand side of (5) with respect to $h_{i} \in[0, \delta], 1 \leqslant i \leqslant r+1$, we find that $\Omega_{r+1}(f, \delta)_{p, q, w} \leqslant C_{3} \delta \Omega_{r}\left(f^{\prime}, \delta\right)_{p, q, w}$. If we use the equality $\left(s_{h}-I\right)(f)(x)=h^{-1} \int_{0}^{h} \int_{0}^{t} f^{\prime}(x+s) d s d t$ instead of (4) similarly to (5), we obtain the second inequality of Lemma 5 in the case $k=1$. The general case easily follows from this one. 
Lemma 6 is proved in [19].

Lemma 6. Let $l \in \mathbb{N}, q>0$. Then there exists $C=C(l, q)$ independent of $n$, such that

$$
\sum_{k=0}^{n}\left(\begin{array}{l}
n \\
k
\end{array}\right) \frac{q^{n-k}}{(k+1)^{l}} \leqslant c \frac{(q+1)^{n}}{(n+1)^{l}}, \quad n \in \mathbb{N} .
$$

Lemma 7 was established by Iofina [8].

Lemma 7. Let $\gamma_{n}(t)=e^{-t} \sum_{k=0}^{n} t^{k} / k !, t \geqslant 1$. Then $\gamma_{[t]}(t) \geqslant C>0$, where $[t]$ is the integer part of $t$.

4. Direct and inverse approximation theorems. As usually, $A(t) \asymp B(t), t \in T$, means that there exist $C_{1}, C_{2}>0$, such that $C_{1} A(t) \leqslant B(t) \leqslant C_{2} A(t), t \in T$.

Theorem 1. Let $1<p, q<\infty, w \in A_{p}(\mathbb{T}), r \in \mathbb{N}, f \in W_{p, q, w}^{r}$. Then

$$
\Omega_{r}(f, t)_{p, q, w} \asymp K\left(f, t^{r}, L_{w}^{p, q}, W_{p, q, w}^{r}\right), \quad t \in[0,2 \pi] .
$$

Proof. By the uniform boundedness of the Steklov operators $s_{h}$ in $L_{w}^{p, q}$ and Lemma 5 for $g \in W_{p(\cdot), 2 \pi}^{r}$, we have

$$
\begin{aligned}
\Omega_{r}(f, t)_{p, q, w} \leqslant \Omega_{r}(f-g, t)_{p, q, w}+ & \Omega_{r}(g, t)_{p, q, w} \leqslant \\
& \leqslant C_{1}\|f-g\|_{p, q, w}+C_{2} t^{r}\left\|g^{(r)}\right\|_{p, q, w} .
\end{aligned}
$$

Taking the infimum in the right-hand side of (6) over $g \in W_{p, q, w}^{r}$, we obtain

$$
\Omega_{r}(f, t)_{p, q, w} \leqslant \max \left(C_{1}, C_{2}\right) K\left(f, t^{r}, L_{w}^{p, q}, W_{p, q, w}^{r}\right) .
$$

For the converse inequality, we use the operator

$$
\Theta_{z}(f)(x)=\frac{2}{z^{2}} \int_{0}^{z} \int_{0}^{t} f(x+s) d s d t .
$$

In [18], it is proved that

$$
\left(\Theta_{z}^{k}(f)\right)^{(k)}(x)=\frac{2^{k}}{z^{k}}\left[s_{z}-I\right]^{k}(f)(x) .
$$


Similar to the proof of Lemma 5 , by Lemma 4 we have

$$
\begin{aligned}
&\left\|\Theta_{z}(f)\right\|_{p, q, w} \leqslant \frac{2}{z^{2}} \int_{0}^{z} t \| t^{-1} \int_{0}^{t} f(x+s) d s \|_{p, q, w} d t \leqslant \\
& \leqslant C_{1}\|f\|_{p, q, w} \frac{2}{z^{2}} \int_{0}^{z} t d t=C_{1}\|f\|_{p, q, w} .
\end{aligned}
$$

For the operators $A_{z}^{[r]}=I-\left(I-\Theta_{z}^{r}\right)^{r}$ and $U_{j}=\Theta_{z}^{r(r-j-1)}$ by (7) we find that

$$
\begin{aligned}
& \left\|\left(A_{z}^{[r]}(f)\right)^{(r)}\right\|_{p, q, w} \leqslant \sum_{j=0}^{r-1}\left(\begin{array}{c}
r \\
j
\end{array}\right)\left\|\left(\Theta_{z}^{r(r-j)}(f)\right)^{(r)}\right\|_{p, q, w}= \\
& =\sum_{j=0}^{r-1}\left(\begin{array}{l}
r \\
j
\end{array}\right)\left\|\left(\Theta_{z}^{r}\left(U_{j}(f)\right)\right)^{(r)}\right\|_{p, q, w}=\sum_{j=0}^{r-1}\left(\begin{array}{l}
r \\
j
\end{array}\right) \frac{2^{r}}{z^{r}}\left\|\left(I-s_{z}\right)^{r}\left[U_{j}(f)\right]\right\|_{p, q, w} .
\end{aligned}
$$

Since $s_{h}$ and $\Theta_{z}$ commute, by (8) we obtain

$$
\begin{aligned}
\left\|\left(A_{z}^{[r]}(f)\right)^{(r)}\right\|_{p, q, w} & \leqslant 2^{r} \sum_{j=0}^{r-1}\left(\begin{array}{l}
r \\
j
\end{array}\right) z^{-r}\left\|U_{j}\left[\left(I-s_{z}\right)^{r}(f)\right]\right\|_{p, q, w} \leqslant \\
& \leqslant C_{2} z^{-r}\left\|\left(I-s_{z}\right)^{r}(f)\right\|_{p, q, w} \leqslant C_{2} z^{-r} \Omega_{r}(f, z)_{p, q, w} .
\end{aligned}
$$

Using (8) and Lemma 4, we have for $g \in L_{2 \pi}^{p(\cdot)}$ :

$$
\left\|\left(I-\Theta_{z}^{r}\right)(g)\right\|_{p, q, w} \leqslant C_{3} \sup _{0 \leqslant t \leqslant z}\left\|\left(I-s_{z}\right)(g)\right\|_{p, q, w} .
$$

(see similar arguments in $[18,(4.6)]$ ). Using the equality $I-A_{z}^{[r]}=\left(I-\Theta_{z}^{r}\right)^{r}$ and applying (10) $r$ times, we obtain

$$
\left\|f-A_{z}^{[r]}(f)\right\|_{p, q, w} \leqslant C_{3}^{r} \Omega_{r}(f, z)_{p, q, w} .
$$

From (9) and (11) we deduce

$$
\left\|f-A_{z}^{[r]}(f)\right\|_{p, q, w}+z^{r}\left\|\left(A_{z}^{[r]}(f)\right)^{(r)}\right\|_{p, q, w} \leqslant C_{4} \Omega_{r}(f, z)_{p, q, w}
$$

where $A_{z}^{[r]}(f) \in W_{p(\cdot), 2 \pi}^{r}$. Thus, $K\left(f, z^{r}, L_{w}^{p, q}, W_{p, q, w}^{r}\right) \leqslant C_{4} \Omega_{r}(f, z)_{p(\cdot)}$, and the proof of Theorem 1 is complete. 
Now we compare our modulus of smoothness with $\Omega_{r}^{*}(f, \delta)_{p, q, w}$ used in [12] and [1].

Corollary 1. Let $1<p, q<\infty, w \in A_{p}(\mathbb{T}), r \in \mathbb{N}$. Then

$$
\Omega_{2 r}(f, \delta)_{p, q, w} \asymp \Omega_{r}^{*}(f, \delta)_{p, q, w}, \quad \delta \in[0,2 \pi] .
$$

Proof. In [1] it is proved that, under conditions of the Corollary,

$$
\Omega_{r}^{*}(f, \delta)_{p, q, w} \asymp K\left(f, \delta^{r}, L_{w}^{p, q}, W_{p, q, w}^{2 r}\right), \quad \delta \in[0,2 \pi] .
$$

Combining this result with Theorem 1, we obtain (12).

Theorem 2. Let $1<p, q<\infty, w \in A_{p}(\mathbb{T}), r \in \mathbb{N}$. Then

$$
E_{n}(f)_{p, q, w} \leqslant C \Omega_{r}\left(f,(n+1)^{-1}\right)_{p, q, w}, \quad n \in \mathbb{Z}_{+} .
$$

Proof. For $n \in \mathbb{Z}_{+}$we choose a function $g \in W_{p, q, w}^{r}$, such that

$$
\|f-g\|)_{p, q, w}+(n+1)^{-r}\left\|g^{(r)}\right\|_{p, q, w} \leqslant 2 K\left(f,(n+1)^{-r}, L_{w}^{p, q}, W_{p, q, w}^{r}\right) .
$$

By Lemma 3 and Theorem 1, we have, for $n \in \mathbb{Z}_{+}$:

$$
\begin{aligned}
E_{n}(f)_{p, q, w} \leqslant & E_{n}(f-g)_{p, q, w}+E_{n}(g)_{p, q, w} \leqslant \\
& \leqslant C_{1}\left(\|f-g\|_{p, q, w}+\left\|g^{(r)}\right\|_{p, q, w}(n+1)^{-r}\right) \leqslant \\
\leqslant & 2 C_{1} K\left(f,(n+1)^{-r}, L_{w}^{p, q}, W_{p, q, w}^{r}\right) \leqslant C_{2} \Omega_{r}\left(f,(n+1)^{-1}\right)_{p, q, w} .
\end{aligned}
$$

Theorem 3. Let $1<p, q<\infty, w \in A_{p}(\mathbb{T}), r \in \mathbb{N}$. Then

$$
\Omega_{r}\left(f, n^{-1}\right)_{p, q, w} \leqslant C n^{-r} \sum_{k=1}^{n} k^{r-1} E_{k-1}(f)_{p, q, w}, \quad n \in \mathbb{N} .
$$

Proof. Let $t_{k} \in T_{k}$ be the polynomial of the best approximation for $f \in L_{w}^{p, q}, k \in \mathbb{Z}_{+}$. Using Lemma 5 and Lemma 2, we obtain

$$
\begin{gathered}
\Omega_{r}\left(f, n^{-1}\right)_{p, q, w} \leqslant \Omega_{r}\left(f-t_{2^{m}, n^{-1}}\right)_{p, q, w}+\Omega_{r}\left(t_{2^{m}}, n^{-1}\right)_{p, q, w} \leqslant \\
\leqslant C_{1}\left(\left\|f-t_{2^{m}}\right\|_{p, q, w}+n^{-r}\left\|t_{2^{m}}^{(r)}\right\|_{p, q, w}\right) \leqslant \\
\leqslant C_{2}\left[E_{2^{m}}(f)_{p, q, w}+n^{-r}\left(\left\|t_{1}^{(r)}-t_{0}^{(r)}\right\|_{p, q, w}+\sum_{i=0}^{m-1}\left\|t_{2^{i+1}}^{(r)}-t_{2^{2}}^{(r)}\right\|_{p, q, w}\right)\right] \leqslant
\end{gathered}
$$




$$
\begin{gathered}
\leqslant C_{3}\left[E_{2^{m}}(f)_{p, q, w}+n^{-r}\left(\left\|t_{1}-t_{0}\right\|_{p, q, w}+\sum_{i=0}^{m-1} 2^{i r}\left\|t_{2^{i+1}}-t_{2^{i}}\right\|_{p, q, w}\right)\right] \leqslant \\
\leqslant C_{4}\left[E_{2^{m}}(f)_{p, q, w}+n^{-r}\left(E_{0}(f)_{p, q, w}+\sum_{i=0}^{m-1} 2^{i r} E_{2^{i}}(f)_{p, q, w}\right)\right] \leqslant \\
\leqslant C_{5} \sum_{k=1}^{2^{m}} k^{r-1} E_{k-1}(f)_{p, q, w}, \quad n \in \mathbb{N} .
\end{gathered}
$$

If $n \in \mathbb{N}$ is fixed and $2^{m} \leqslant n<2^{m+1}, m \in \mathbb{Z}_{+}$, then (13) easily follows from the last inequality.

If $\omega$ is increasing and continuous on $[0 ; 2 \pi], \omega(0)=0$, then $\omega \in \Phi$. A function $\omega \in \Phi$ belongs to the Bary-Stechkin class $B_{\alpha}, \alpha>0$, if $\sum_{k=1}^{n} k^{\alpha-1} \omega\left(k^{-1}\right)=O\left(n^{\alpha} \omega\left(n^{-1}\right)\right), n \in \mathbb{N}($ see [4]).

Corollary 1. Let $1<p, q<\infty, w \in A_{p}(\mathbb{T}), r \in \mathbb{N}$ and $\omega \in B_{r}$. Then, the conditions $E_{n}(f)_{p, q, w}=O\left(\omega\left((n+1)^{-1}\right)\right), n \in \mathbb{Z}_{+}$, and $\Omega_{r}(f, \delta)_{p, q, w}=O(\omega(\delta)), \delta \in[0,2 \pi]$, are equivalent.

Remark 1. The converse inequality from Theorem 3 has the same form as the classical converse approximation theorem (see [6, Ch. 7, Theorem 3.1]), while (see Corollary 1)

$$
\Omega_{r}^{*}\left(f, n^{-1}\right)_{p, q, w} \leqslant C n^{-2 r} \sum_{k=1}^{n} k^{2 r-1} E_{k-1}(f)_{p, q, w}, \quad n \in \mathbb{N} .
$$

\section{Approximation by the Borel and Euler means.}

Theorem 4. Let $1<p, q<\infty, w \in A_{p}(\mathbb{T}), r \in \mathbb{N}, t>0$ and $f \in L_{w}^{p, q}$. Then

$$
\begin{gathered}
\left\|f-e_{n}^{t}(f)\right\|_{p, q, w} \leqslant C \Omega_{r}\left(f, n^{-1}\right)_{p, q, w}, \quad n \in \mathbb{N}, \\
\left\|f-e_{n}^{t}(f)\right\|_{p, q, w} \leqslant C n^{-r} \sum_{k=1}^{n} k^{r-1} E_{k-1}(f)_{p, q, w}, \quad n \in \mathbb{N} .
\end{gathered}
$$

Proof. By the definition of the Euler means, Lemma 1, and Theorem 2, we obtain

$$
\left\|f-e_{n}^{t}(f)\right\|_{p, q, w}=(1+t)^{-n}\left\|\sum_{j=0}^{n}\left(\begin{array}{c}
n \\
j
\end{array}\right) t^{n-j}\left(f-S_{j}(f)\right)\right\|_{p, q, w} \leqslant
$$




$$
\begin{aligned}
& \leqslant \frac{C_{1}}{(1+t)^{n}} \sum_{j=0}^{n}\left(\begin{array}{l}
n \\
j
\end{array}\right) t^{n-j} E_{j}(f)_{p, q, w} \leqslant \\
& \leqslant \frac{C_{2}}{(1+t)^{n}} \sum_{j=0}^{n}\left(\begin{array}{l}
n \\
j
\end{array}\right) t^{n-j} \Omega_{r}\left(f,(j+1)^{-1}\right)_{p, q, w} .
\end{aligned}
$$

Due to Theorem 1, we have the property

$$
\Omega_{r}(f, \lambda \delta)_{p, q, w} \leqslant C(\lambda+1)^{r} \Omega_{r}(f, \delta)_{p, q, w}, \quad \lambda>0 .
$$

By this property and Lemma 6, we find that

$\left\|f-e_{n}^{t}(f)\right\|_{p, q, w} \leqslant \frac{C_{2}}{(1+t)^{n}} \sum_{j=0}^{n}\left(\begin{array}{c}n \\ j\end{array}\right) t^{n-j}\left(\frac{n+1}{j+1}+1\right)^{r} \Omega_{r}\left(f, \frac{1}{n+1}\right)_{p, q, w} \leqslant$ $\leqslant \frac{C_{3}}{(1+t)^{n}}(n+1)^{r} \Omega_{r}\left(f, \frac{1}{n+1}\right)_{p, q, w} \sum_{j=0}^{n}\left(\begin{array}{c}n \\ j\end{array}\right) \frac{t^{n-j}}{(j+1)^{r}} \leqslant C_{4} \Omega_{r}\left(f, \frac{1}{n+1}\right)_{p, q, w}$ and (14) is proved. The inequality (15) follows from (14) and Theorem 3.

Theorem 5. Let $1<p, q<\infty, w \in A_{p}(\mathbb{T}), r \in \mathbb{N}, t \geqslant 1$ and $f \in L_{w}^{p, q}$. Then

$$
\left\|f-B_{t}(f)\right\|_{p, q, w} \leqslant C \sum_{k=0}^{[t]} \frac{t^{k}}{k !} e^{-t} E_{k}(f)_{p, q, w},
$$

where $[t]$ is the integer part of $t$.

Proof. Let $\tau_{n} \in T_{n}$ be such that $\left\|f-\tau_{n}\right\|_{p, q, w}=E_{n}(f)_{p, q, w}$. Then, by Lemma 1,

$$
\begin{gathered}
\left\|f-B_{t}(f)\right\|_{p, q, w} \leqslant\left\|B_{t}\left(\tau_{n}\right)-B_{t}(f)\right\|_{p, q, w}+\left\|B_{t}\left(\tau_{n}\right)-\tau_{n}\right\|_{p, q, w}+ \\
+\left\|\tau_{n}-f\right\|_{p, q, w} \leqslant e^{-t} \sum_{k=0}^{\infty} \frac{t^{k}}{k !}\left\|S_{k}\left(f-\tau_{n}\right)\right\|_{p, q, w}+\left\|B_{t}\left(\tau_{n}\right)-\tau_{n}\right\|_{p, q, w}+ \\
+\left\|\tau_{n}-f\right\|_{p, q, w} \leqslant C_{1} E_{n}(f)_{p, q, w}+\left\|B_{t}\left(\tau_{n}\right)-\tau_{n}\right\|_{p, q, w} .
\end{gathered}
$$

Now,

$$
\left\|B_{t}\left(\tau_{n}\right)-\tau_{n}\right\|_{p, q, w}=\left\|\sum_{k=0}^{n-1} \frac{t^{k} e^{-t}}{k !}\left(S_{k}\left(\tau_{n}\right)-\tau_{n}\right)\right\|_{p, q, w} \leqslant
$$




$$
\leqslant C_{2} \sum_{k=0}^{n-1} \frac{t^{k} e^{-t}}{k !} E_{k}\left(\tau_{n}\right)_{p, q, w}
$$

By the definition of $\tau_{n}$,

$$
E_{k}\left(\tau_{n}\right)_{p, q, w} \leqslant E_{k}\left(f-\tau_{n}\right)_{p, q, w}+E_{k}(f)_{p, q, w} \leqslant 2 E_{k}(f)_{p, q, w} .
$$

Using the previous inequaities and Lemma 7 and taking $n=[t]$, we obtain

$$
\begin{aligned}
\left\|f-B_{t}(f)\right\|_{p, q, w} \leqslant C_{1} E_{[t]}(f)_{p, q, w}+2 C_{2} \sum_{k=0}^{[t]-1} \frac{t^{k} e^{-t}}{k !} E_{k}(f)_{p, q, w} \leqslant \\
\leqslant C_{3} \sum_{k=0}^{[t]} \frac{t^{k} e^{-t}}{k !} E_{k}(f)_{p, q, w} .
\end{aligned}
$$

Let us show that the estimate of Theorem 5 gives a clear result on some subclasses of $L_{w}^{p, q}$.

Corollary 1. Let $1<p, q<\infty, w \in A_{p}(\mathbb{T}), r \in \mathbb{N}, t \geqslant 1$ and $f \in L_{w}^{p, q}$. If $E_{n}(f)_{p, q, w}=O\left((n+1)^{-\alpha}\right), n \in \mathbb{Z}_{+}$, or $\Omega(f, \delta)_{p, q, w}=O\left(\delta^{\alpha}\right), \delta \in[0,2 \pi]$, then

$$
\left\|f-B_{t}(f)\right\|_{p, q, w} \leqslant C t^{-\alpha}, \quad t \geqslant 1 .
$$

Proof. Under conditions of Corollary 1, we have, by Theorem 5 and Theorem 2:

$$
\left\|f-B_{t}(f)\right\|_{p, q, w} \leqslant C_{1} \sum_{k=0}^{[t]} \frac{t^{k} e^{-t}}{k !(k+1)^{\alpha}}=C_{1}(t+1)^{-\alpha} \sum_{k=0}^{[t]} \frac{t^{k} e^{-t}}{k !}\left(\frac{t+1}{k+1}\right)^{\alpha} .
$$

For $m=[\alpha]+1$ and $0 \leqslant k \leqslant[t]$, we have

$$
\left(\frac{t+1}{k+1}\right)^{\alpha} \leqslant\left(\frac{t+1}{k+1}\right)^{m} \leqslant \frac{2^{m} t^{m}}{(k+1)^{m}}
$$

and

$$
\left\|f-B_{t}(f)\right\|_{p, q, w} \leqslant C_{2}(t+1)^{-\alpha} e^{-t} \sum_{k=0}^{[t]} \frac{t^{k+m}}{k !(k+1)^{m}} \leqslant
$$




$$
\begin{gathered}
\leqslant \frac{C_{2}}{(t+1)^{\alpha} e^{t}} \sum_{k=0}^{\infty} \frac{t^{k+m}}{(k+m) !} \frac{(k+1) \ldots(k+m)}{(k+1)^{m}} \leqslant \\
\leqslant \frac{C_{2} m !}{(t+1)^{\alpha} e^{t}} \sum_{k=0}^{\infty} \frac{t^{k+m}}{(k+m) !} \leqslant \frac{C_{3}}{(t+1)^{\alpha}}
\end{gathered}
$$

Acknowledgment. Supported by the Ministry of science and education of the Russian Federation in the framework of the basic part of the scientific research state task, project FSRR-2020-0006

\section{References}

[1] Akgün R., Yildirir Y. E. Jackson-Stechkin type inequality in weighted Lorentz spaces. Math. Inequal. Appl., 2015, vol. 18, no. 4, pp. 1283-1293. DOI: https://dx.doi.org/10.7153/mia-18-100

[2] Akgün R., Yildirir Y. E. Improved direct and converse theorems in weighted Lorentz spaces. Bull. Belg. Math. Soc. Simon Stevin, 2016, vol. 23, no. 2, pp. 247-262. DOI: https://doi.org/10.36045/bbms/1464710117

[3] Bary N. K. A treatise on trigonometric series. Pergamon Press, New York, 1964.

[4] Bary N. K., Stechkin S. B. Best approximations and differential properties of two conjugate functions. Trudy Moskov. Mat. Obshch., 1956, vol. 5, pp. 483-522 (in Russian).

[5] Chang H. M., Hunt R. A., Kurtz D. S. The Hardy-Littlewood maximal function on $L(p, q)$ spaces with weights. Indiana Univ. Math. J., 1982, vol. 31, no. 1 , pp. $109-120$.

DOI: https://doi.org/10.1512/iumj.1982.31.31012

[6] DeVore R. A., Lorentz G. G. Constructive approximation. Springer, BerlinHeidelberg-New York, 1993.

[7] Hardy G. H. Divergent series. Oxford Univ. Press, Oxford, 1949.

[8] Iofina T. V. Approximation of functions by Borel means of Fourier series with respect to multiplicative systems. Izv. Saratov Univ. (N. S.), Ser. Math. Mech. Inform., 2011, vol. 11, no. 3(1), pp. 15-21. (in Russian)

[9] Israfilov D., Kokilashvili V., Samko S. Approximation in weighted Lebesgue and Smirnov spaces with variable exponents. Proc. A.Razmadze Math. Inst., 2007, vol. 143, pp. 25-35. 
[10] Israfilov D. M., Testici A. Some inverse and simultaneous approximation theorems in weighted variable exponent Lebesgue spaces. Analysis Math., 2018, vol. 44, no. 4, pp. 475-492. (in Russian)

DOI: https://doi.org/10.1007/s10476-018-0403-x

[11] Kokilashvili V., Krbec M. Weighted inequalities in Lorentz and Orlicz spaces, World Scientific Publishing Co. Inc. River Edge, NJ, 1991.

[12] Kokilashvili V., Yildirir Y. E. On the approximation by trigonometric polynomials in weighted Lorentz spaces. J. Funct. Spaces Appl., 2010, vol. 8, no. 1 , pp. $67-86$.

[13] Ky N. X. Moduli of mean smoothness and approximation with $A_{p}$-weights. Annales Univ. Sci. Budapest, 1997, vol. 40, 37-48.

[14] Ky N. X. An Alexits lemma and its applications in approximation theory. Functions, Series, Operators. Budapest, 2002 (L. Leindler, F. Schipp, J. Szabados, eds.), pp. 287-296.

[15] Lorentz G. G. On the theory of spaces $\Lambda$. Pacific J. Math., 1951, vol. 1, no. 3, pp. 411-429.

[16] Muckenhoupt B. Weighted norm inequalities for the Hardy maximal function. Trans. Amer. Math. Soc., 1972, vol. 165, pp. 207-226.

DOI: https://doi.org/10.1090/S0002-9947-1972-0293384-6

[17] Sharapudinov I. I. Approximation of functions in $L_{2 \pi}^{p(x)}$ by trigonometric polynomials. Izv. Ross. Akad. Nauk Ser. Mat., 2013, vol. 77, no. 2, pp. $197-$ 224 (in Russian). Translated in Izv. Math., vol. 77, no. 2, pp. 407-434.

DOI: https://doi.org/10.4213/im7808

[18] Volosivets S. S. Approximation of functions and their conjugates in variable Lebesgue spaces. Sbornik: Math., 2017, vol. 208, no. 1, pp. 44-59. Transl. from Mat. sbornik, 2017, vol. 208, no. 1, pp. 48-64.

DOI: https://doi.org/10.1070/SM8636

[19] Volosivets S. S., Tyuleneva A. A. Approximation of functions and their conjugates in $L^{p}$ and uniform metric by Euler means. Demonstr. Math., 2018, vol. 51, no. 1, pp. $141-150$.

DOI: https://doi.org/10.1515/dema-2018-0013

[20] Yildirir Y. E., Israfilov D. M. Approximation theorems in weighted Lorentz spaces. Carpathian J. Math., 2010, vol. 26, no. 1, pp. 108-119.

[21] Yurt H., Guven A. Multivariate Approximation theorems in weighted Lorentz spaces. Mediterr. J. Math., 2015, vol. 12, no. 3, pp. 863-876.

DOI: https://doi.org/10.1007/s00009-014-0446-6 
Received August 17, 2020.

In revised form, November 30, 2020.

Accepted December 09, 2020.

Published online December 22, 2020.

Saratov State University

83 Astrakhanskaya St., Saratov 410012, Russia

E-mail: VolosivetsSS@mail.ru 